\title{
Rough Set Approach for Analyzing the Effect of Viscoelastic and Micropolar Parameters on Hiemenz Flow in Hydromagnetics
}

\author{
Hossam A. Nabwey ${ }^{1,2}$ \\ ${ }^{I}$ Department of Mathematics, College of Science and Humanities in Al-Kharj, Prince Sattam bin Abdulaziz University, \\ Al-Kharj 11942, Saudi Arabia. \\ ${ }^{2}$ Department of Basic Engineering Science, Faculty of Engineering, Menoufia University, Shebin El-Kom,32511, Egypt.
}

ORCID: 0000-0002-7167-3822

\begin{abstract}
This research describes the hydromagnetic problem of two dimensional Hiemenz flow for a micropolar, viscoelastic, incompressible, viscous, electrically conducting fluid, impinging perpendicularly onto a plane in the presence of a transverse magnetic field. An approach based on the rough set theory is introduced where the mathematical model which describes the problem is first transformed into a dimensionless form. Then it is solved by using the Runge-Kutta numerical integration procedure in conjunction with shooting technique. Finally a set of maximally generalized decision rules (classification rules) are generated by using rough sets methodology.
\end{abstract}

Keywords: Viscoelastic fluids; Micropolar fluids; Hiemenz Flow; Rough set theory; feature selection; Hydromagnetics.

\section{NOMENCLATURE}

$\bar{u}$ and $\bar{v} \quad$ Velocity components along $x$ and $y$ axes

$\mathrm{X}$ and $\mathrm{y}$ Dimensionless velocity component in the $\mathrm{X}$ - and y-direction

$\mathrm{N} \quad$ angular velocity

$v \quad$ Kinematic viscosity

$\sigma \quad$ electrical conductivity of fluid

$\mathrm{K}^{*} \quad$ weissenberg number

B induced magnetic field

a constant

$\mu \quad$ Dynamic viscosity

$\mathrm{K} \quad$ vortex viscosity

$\gamma \quad$ spin gradient viscosity

j microinertia per unit mass

$\mathrm{k}_{0} \quad$ viscoelastic parameter

M Hartman Number

\section{1) INTRODUCTION}

In recent past the attention of many scientists was attracted to viscoelastic fluids due to application of this kind of fluids in industrial engineering, chemical industries, biomedical engineering, paints, polymers and technological applications since this type of fluids retains old distortions and its new behavior depends on previous distortions due to its "elastic" nature. Beard and Walters [1] developed the first model which describe and simulate the viscous fluids then many scientists and engineers studied and analyzed the flow and heat transfer characteristics of viscoelastic fluids as a type of nonNewtonian fluids [2-10].

Hiemenz was the first one who studied the two dimensional flow of a fluid near a stagnation point and show that the governing equations which describe the flow can be reduced to an ordinary differential equation with the aid of similarity transformation [11] then Several studies were elaborated by researchers to study Hiemenz flow in different ways to include various physical effects in hydromagnetics [12-14]. Also, Micropolar fluids are introduced by Eringen [15] and he characterized the structure of these fluids and define it physically as it consist of rigid, randomly oriented (or spherical) particles suspended in a viscous medium and theses particles can rotate with their own spins and microrotations, since the deformation of fluid particles is ignored. Then Eringen [16] extended his investigation of micropolar elasticity and many researchers and engineers focus their efforts in studying micropolar fluids as it has a great role in industrial applications Examples include exotic lubricants, food industry, biological and bio-medical sciences. For excellent review see [17-19].

The problem of reducing has been investigated for many numerous applications in different fields, since the irrelevant and redundant features in the dataset lead to low accuracy. There are two main approaches to reduce the input dimensionality, namely feature extraction and feature selection. Rough set theory was used as a tool to reduce the dimensionality as well as dealing with uncertainty in datasets. Many heuristic algorithms are proposed based on rough set theory, also numerous approached based on rough set theory and other theory are investigated to extract decision rules and reduce the dimensionality of dataset [20-30]. 
In this paper, we consider the effect of a transverse magnetic field on the Hiemenz flow (the two dimensional flow near a stagnation point) of micropolar viscoelastic fluids. The governing Equations are solved by using the Runge-Kutta numerical integration procedure in conjunction with shooting technique. We present numerical results for a range of values of the Hartman number, of the viscoelastic parameter, and of the material properties of the fluid. Besides, the outcomes are elaborated graphically for involved variables.

\section{2) MATHEMATICAL FLOW MODEL}

Let us consider two-dimensional flow of a viscous, incompressible, electrically conducting, micropolar, viscoelastic fluid impinging perpendicularly onto a plane directed along the $x$-axis, as shown in Fig. 1. The flow is embedded in a uniform magnetic field of constant strength $H_{0}$.

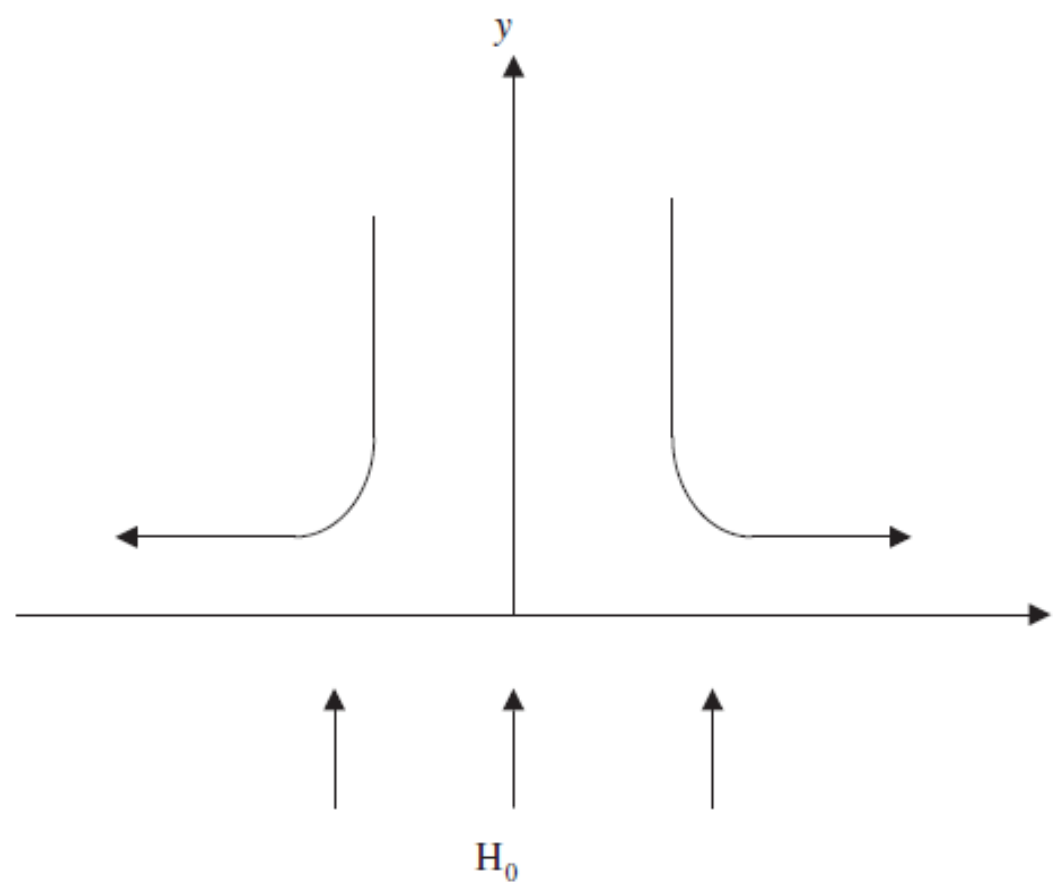

Fig. 1. Flow model and coordinate system

The governing equations which describe the mathematical model for this problem take the form [33]:

$$
\begin{aligned}
& \frac{\partial \bar{u}}{\partial x}+\frac{\partial \bar{v}}{\partial y}=0, \\
& \bar{u} \frac{\partial \bar{u}}{\partial x}+\bar{v} \frac{\partial \bar{u}}{\partial y}=a^{2} x+\left(v+\frac{k}{\rho}\right) \frac{\partial^{2} \bar{u}}{\partial y^{2}}+\frac{k}{\rho} \frac{\partial N}{\partial y}+ \\
& \frac{\sigma B^{2}}{\rho}(a x-\bar{u})-k^{*}\left(\bar{u} \frac{\partial^{3} \bar{u}}{\partial x y^{2}}+\bar{v} \frac{\partial^{3} \bar{u}}{\partial y^{3}}+\frac{\partial \bar{u}}{\partial x} \frac{\partial^{2} \bar{u}}{\partial y^{2}}-\frac{\partial \bar{u}}{\partial y} \frac{\partial^{2} \bar{u}}{\partial x \partial y}\right) \\
& \bar{u} \frac{\partial N}{\partial x}+\bar{v} \frac{\partial N}{\partial y}=\frac{\gamma}{\rho j} \frac{\partial^{2} N}{\partial y^{2}}-\frac{k j}{\rho j}\left(2 N+\frac{\partial \bar{u}}{\partial y}\right),
\end{aligned}
$$

According to [33] Boundary conditions for the for stagnation-point flow are as follows:

$$
\begin{array}{lll}
y=0: & \bar{u}=0, & \bar{v}=0, \\
y \rightarrow \infty: & \quad \bar{u}=a x, & N=0
\end{array}
$$


The partial differential conservation equations (1)-(4) are thereby converted into dimensionless system by defining the stream function $\psi$ as:

$$
\bar{u}=\frac{\partial \psi}{\partial y} \quad \bar{v}=-\frac{\partial \psi}{\partial x}
$$

And the following non-dimensional variables are introduced:

$$
\eta=\sqrt{\frac{a}{\bar{u}}} y, \quad \psi=\sqrt{a \bar{v}} x f(\eta), \quad N=\sqrt{\frac{a}{\bar{v}}} \operatorname{axg}(\eta)
$$

Also to facilitate numerical solutions for low values of the viscoelastic parameter $k_{0}$, the following formalization expressions are used:

$$
\begin{aligned}
& f=f_{0}+k_{0} f_{1}+k_{0}^{2} f_{2}+\ldots \ldots \ldots \ldots \ldots \\
& g=g_{0}+k_{0} g_{1}+k_{0}^{2} g_{2}+\ldots \ldots \ldots \ldots \ldots
\end{aligned}
$$

The final mathematical model was obtained as:

$$
\begin{aligned}
& (1+\Delta) f_{0}^{\prime \prime \prime}+\Delta g_{0}^{\prime}+f_{0} f_{0}^{\prime \prime}+1-f_{0}^{\prime 2}+M^{2}\left(1-f_{0}^{\prime}\right)=0 \\
& \lambda_{0} g_{0}^{\prime \prime}-\Delta \cdot B_{1}\left(2 g_{0}+f_{0}^{\prime \prime}\right)-f_{0}^{\prime} g_{0}+g_{0}^{\prime} f_{0}=0 \\
& (1+\Delta) f_{1}^{\prime \prime \prime}+\Delta g_{1}^{\prime}+f_{0} f_{1}^{\prime \prime}+f_{1} f_{0}^{\prime \prime}-2 f_{0}^{\prime} f_{1}^{\prime}-M^{2} f_{1}^{\prime}-\left(2 f_{0}^{\prime} f_{1}^{\prime \prime \prime}-f_{0} f_{0}^{i v}-f_{0}^{\prime 2}\right)=0 \\
& \lambda g_{1}^{\prime \prime}-\Delta \cdot B_{1}\left(2 g_{1}+f_{1}^{\prime \prime}\right)-f_{0}^{\prime} g_{1}-f_{1}^{\prime} g_{0}+g_{0}^{\prime} f_{1}+g_{1}^{\prime} f_{0}=0 \\
& (1+\Delta) f_{2}^{\prime \prime \prime}+\Delta g_{2}^{\prime}+f_{0} f_{2}^{\prime \prime}+f_{2} f_{0}^{\prime \prime}+f_{1} f_{1}^{\prime \prime}-2 f_{0}^{\prime} f_{2}^{\prime}-f_{1}^{\prime 2}-M^{2} f_{2}^{\prime} \\
& \quad-\left(2 f_{0}^{\prime} f_{1}^{\prime \prime \prime}+2 f_{1}^{\prime} f_{0}^{\prime \prime \prime}-f_{0} f_{1}^{i v}-f_{1} f_{0}^{i v}-2 f_{0}^{\prime \prime} f_{1}^{\prime \prime}\right)=0 \\
& \lambda g_{2}^{\prime \prime}-\Delta . B_{1}\left(2 g_{2}+f_{2}^{\prime \prime}\right)-f_{0}^{\prime} g_{2}-f_{1}^{\prime} g_{1}-f_{2}^{\prime} g_{0}+g_{0}^{\prime} f_{2}+g_{1}^{\prime} f_{1}+g_{2}^{\prime} f_{0}=0
\end{aligned}
$$

Subject to the boundary conditions:

$$
\begin{aligned}
& f_{0}(0)=f_{1}(0)=f_{2}(0)=f_{0}^{\prime}(0)=f_{1}^{\prime}(0)=f_{2}^{\prime}(0)=0 \\
& f_{0}^{\prime}(\infty)=1, \quad f_{1}^{\prime}(\infty)=f_{2}^{\prime}(\infty)=0 \\
& g_{0}(0)=-m f_{0}^{\prime \prime}(0), \quad g_{1}(0)=-m f_{1}^{\prime \prime}(0), \quad g_{2}(0)=-m f_{0}^{\prime \prime}(0) \\
& g_{0}(\infty)=g_{1}(\infty)=g_{2}(\infty)=0
\end{aligned}
$$

\section{3) ANALYSIS}

The system of equations (8)-(13) subject to the boundary condition in (4) was solved by the aid of Runge-Kutta numerical-integration procedure in conjunction with a shooting technique. The results of these calculations are divided into two parts. The first part represents the case of viscoelastic fluids where the surface values $f^{\prime \prime}(0)$ of the velocity gradient at $\Delta=0$ are shown in table 1 . While the 
International Journal of Engineering Research and Technology. ISSN 0974-3154, Volume 13, Number 1 (2020), pp. 170-180

(C) International Research Publication House. https://dx.doi.org/10.37624/IJERT/13.1.2020.170-180

second part represents the case of micropolar viscoelastic fluid, where the surface values $f^{\prime \prime}(0)$ of the velocity gradient and the surface values $g^{\prime}(0)$ of the microrotation gradients for various nonzero values of $\Delta$ are shown in table 2 .

Table 1. Values of $f^{\prime \prime}(0)$ at $\Delta=0$ for various values of $\mathrm{M}$ and $\mathrm{k} 0$, for a second-order viscoelastic fluid.

\begin{tabular}{|c|c|c|c|}
\hline $\mathrm{U}$ & M & $\mathrm{K}_{0}$ & $f^{\prime \prime}(0)$ \\
\hline X1 & 0.0 & 0 & 1.23259 \\
\hline X2 & 0.0 & 0.025 & 1.26297 \\
\hline X3 & 0.0 & 0.05 & 1.29336 \\
\hline X4 & 0.2 & 0 & 1.24857 \\
\hline X5 & 0.2 & 0.025 & 1.27933 \\
\hline X6 & 0.2 & 0.05 & 1.31009 \\
\hline $\mathrm{X7}$ & 0.4 & 0 & 1.29537 \\
\hline X8 & 0.4 & 0.025 & 1.32725 \\
\hline X9 & 0.4 & 0.05 & 1.35913 \\
\hline $\mathbf{X 1 0}$ & 0.6 & 0 & 1.36988 \\
\hline X11 & 0.6 & 0.025 & 1.40360 \\
\hline X12 & 0.6 & 0.05 & 1.43732 \\
\hline $\mathrm{X} 13$ & 0.8 & 0 & 1.46798 \\
\hline X14 & 0.8 & 0.025 & 1.50423 \\
\hline $\mathrm{X} 15$ & 0.8 & 0.05 & 1.54048 \\
\hline X16 & 1.0 & 0 & 1.58533 \\
\hline X17 & 1.0 & 0.025 & 1.62475 \\
\hline X18 & 1.0 & 0.05 & 1.66418 \\
\hline X19 & 1.2 & 0 & 1.71804 \\
\hline $\mathbf{X 2 0}$ & 1.2 & 0.025 & 1.76124 \\
\hline $\mathrm{X} 21$ & 1.2 & 0.05 & 1.80444 \\
\hline $\mathbf{X 2 2}$ & 1.4 & 0 & 1.86285 \\
\hline $\mathbf{X} 23$ & 1.4 & 0.025 & 1.91038 \\
\hline $\mathrm{X} 24$ & 1.4 & 0.05 & 1.95790 \\
\hline $\mathbf{X 2 5}$ & 1.6 & 0 & 2.01715 \\
\hline $\mathrm{X} 26$ & 1.6 & 0.025 & 2.06952 \\
\hline $\mathbf{X} 27$ & 1.6 & 0.05 & 2.12189 \\
\hline $\mathbf{X 2 8}$ & 2.0 & 0 & 2.34666 \\
\hline $\mathbf{X 2 9}$ & 2.0 & 0.025 & 2.41013 \\
\hline $\mathbf{X 3 0}$ & 2.0 & 0.05 & 2.47361 \\
\hline X31 & 3.0 & 0 & 3.24095 \\
\hline $\mathbf{X 3 2}$ & 3.0 & 0.025 & 3.33951 \\
\hline $\mathbf{X 3 3}$ & 3.0 & 0.05 & 3.43806 \\
\hline X34 & 5.0 & 0 & 5.14796 \\
\hline X35 & 5.0 & 0.025 & 5.34429 \\
\hline X36 & 5.0 & 0.05 & 5.54062 \\
\hline X37 & 10.0 & 0 & 10.07474 \\
\hline X38 & 10.0 & 0.025 & 10.63314 \\
\hline X39 & 10.0 & 0.05 & 11.25077 \\
\hline
\end{tabular}


Table 2. Values of $f^{\prime \prime}(0)$ and $g^{\prime}(0)$ for various values of $\mathrm{M}, \mathrm{k} 0$ and $\Delta$, for a second-order viscoelastic fluid.

\begin{tabular}{|c|c|c|c|c|c|}
\hline $\mathrm{U}$ & M & $\mathrm{K}_{0}$ & $\Delta$ & $f^{\prime \prime}(0)$ & $-g^{\prime}(0)$ \\
\hline $\mathrm{X1}$ & 0.0 & 0 & 0.5 & 1.00365 & 0.04813 \\
\hline $\mathrm{X} 2$ & 0.0 & 0.025 & 0.5 & 1.01735 & 0.04844 \\
\hline $\mathrm{X3}$ & 0.0 & 0.05 & 0.5 & 1.03106 & 0.044876 \\
\hline $\mathrm{X} 4$ & 0.2 & 0 & 0.5 & 1.01669 & 0.04876 \\
\hline $\mathrm{X5}$ & 0.2 & 0.025 & 0.5 & 1.03056 & 0.04834 \\
\hline X6 & 0.2 & 0.05 & 0.5 & 1.04444 & 0.04865 \\
\hline $\mathrm{X7}$ & 0.4 & 0 & 0.5 & 1.05489 & 0.04897 \\
\hline $\mathrm{X8}$ & 0.4 & 0.025 & 0.5 & 1.06927 & 0.04895 \\
\hline X9 & 0.4 & 0.05 & 0.5 & 1.08365 & 0.04927 \\
\hline X10 & 0.6 & 0 & 0.5 & 1.11573 & 0.04959 \\
\hline X11 & 0.6 & 0.025 & 0.5 & 1.13094 & 0.04989 \\
\hline $\mathrm{X12}$ & 0.6 & 0.05 & 0.5 & 1.14615 & 0.05021 \\
\hline X13 & 0.8 & 0 & 0.5 & 1.19581 & 0.05054 \\
\hline X14 & 0.8 & 0.025 & 0.5 & 1.21216 & 0.05107 \\
\hline X15 & 0.8 & 0.05 & 0.5 & 1.22851 & 0.05140 \\
\hline X16 & 1.0 & 0 & 0.5 & 1.29164 & 0.05173 \\
\hline X17 & 1.0 & 0.025 & 0.5 & 1.30942 & 0.05241 \\
\hline X18 & 1.0 & 0.05 & 0.5 & 1.32720 & 0.05308 \\
\hline X19 & 1.2 & 0 & 0.5 & 1.40001 & 0.05383 \\
\hline $\mathbf{X 2 0}$ & 1.2 & 0.025 & 0.5 & 1.41949 & 0.05418 \\
\hline $\mathrm{X} 21$ & 1.2 & 0.05 & 0.5 & 1.43897 & 0.05452 \\
\hline $\mathbf{X 2 2}$ & 1.4 & 0 & 0.5 & 1.51827 & 0.05529 \\
\hline $\mathrm{X} 23$ & 1.4 & 0.025 & 0.5 & 1.53969 & 0.05564 \\
\hline $\mathrm{X24}$ & 1.4 & 0.05 & 0.5 & 1.56112 & 0.05599 \\
\hline $\mathrm{X} 25$ & 1.6 & 0 & 0.5 & 1.64429 & 0.0673 \\
\hline $\mathrm{X} 26$ & 1.6 & 0.025 & 0.5 & 1.66789 & 0.05710 \\
\hline $\mathbf{X 2 7}$ & 1.6 & 0.05 & 0.5 & 1.69148 & 0.05746 \\
\hline $\mathrm{X} 28$ & 2.0 & 0 & 0.5 & 1.91341 & 0.05950 \\
\hline $\mathbf{X 2 9}$ & 2.0 & 0.025 & 0.5 & 1.94199 & 0.05989 \\
\hline $\mathbf{X 3 0}$ & 2.0 & 0.05 & 0.5 & 1.97057 & 0.06028 \\
\hline X31 & 3.0 & 0 & 0.5 & 2.64380 & 0.06544 \\
\hline X32 & 3.0 & 0.025 & 0.5 & 2.68810 & 0.06588 \\
\hline X33 & 3.0 & 0.05 & 0.5 & 2.73241 & 0.06633 \\
\hline X34 & 5.0 & 0 & 0.5 & 4.20127 & 0.07352 \\
\hline X35 & 5.0 & 0.025 & 0.5 & 4.28933 & 0.07406 \\
\hline X36 & 5.0 & 0.05 & 0.5 & 4.37739 & 0.07459 \\
\hline X37 & 10.0 & 0 & 0.5 & 8.22464 & 0.08223 \\
\hline $\begin{array}{l}\mathrm{X38} \\
\end{array}$ & 10.0 & 0.025 & 0.5 & 8.48811 & 0.08299 \\
\hline X39 & 10.0 & 0.05 & 0.5 & 8.75158 & 0.08375 \\
\hline
\end{tabular}




\begin{tabular}{|c|c|c|c|c|c|}
\hline $\mathrm{U}$ & $\mathrm{M}$ & $\mathrm{K}_{0}$ & $\Delta$ & $f^{\prime \prime}(0)$ & $-g^{\prime}(0)$ \\
\hline $\mathrm{X40}$ & 0.0 & 0 & 1.5 & 0.76688 & 0.12087 \\
\hline $\mathrm{X} 41$ & 0.0 & 0.025 & 1.5 & 0.77215 & 0.12129 \\
\hline $\mathrm{X} 42$ & 0.0 & 0.05 & 1.5 & 0.77741 & 0.12172 \\
\hline $\mathrm{X} 43$ & 0.2 & 0 & 1.5 & 0.77691 & 0.12151 \\
\hline X44 & 0.2 & 0.025 & 1.5 & 0.78224 & 0.12194 \\
\hline $\mathrm{X} 45$ & 0.2 & 0.05 & 1.5 & 0.78757 & 0.12236 \\
\hline $\mathrm{X} 46$ & 0.4 & 0 & 1.5 & 0.80632 & 0.12336 \\
\hline $\mathrm{X} 47$ & 0.4 & 0.025 & 1.5 & 0.81185 & 0.12379 \\
\hline $\mathrm{X} 48$ & 0.4 & 0.05 & 1.5 & 0.81737 & 0.12422 \\
\hline $\mathrm{X} 49$ & 0.6 & 0 & 1.5 & 0.85321 & 0.12622 \\
\hline X50 & 0.6 & 0.025 & 1.5 & 0.85906 & 0.12666 \\
\hline X51 & 0.6 & 0.05 & 1.5 & 0.86490 & 0.12710 \\
\hline $\mathrm{X52}$ & 0.8 & 0 & 1.5 & 0.91502 & 0.12984 \\
\hline X53 & 0.8 & 0.025 & 1.5 & 0.92130 & 0.13029 \\
\hline X54 & 0.8 & 0.05 & 1.5 & 0.92758 & 0.13074 \\
\hline X55 & 1.0 & 0 & 1.5 & 0.98905 & 0.13397 \\
\hline X56 & 1.0 & 0.025 & 1.5 & 0.99587 & 0.13444 \\
\hline X57 & 1.0 & 0.05 & 1.5 & 1.00269 & 0.13490 \\
\hline X58 & 1.2 & 0 & 1.5 & 1.07286 & 0.13838 \\
\hline X59 & 1.2 & 0.025 & 1.5 & 1.08032 & 0.13886 \\
\hline X60 & 1.2 & 0.05 & 1.5 & 1.08778 & 0.13934 \\
\hline X61 & 1.4 & 0 & 1.5 & 1.16438 & 0.14291 \\
\hline $\mathrm{X62}$ & 1.4 & 0.025 & 1.5 & 1.17258 & 0.14341 \\
\hline X63 & 1.4 & 0.05 & 1.5 & 1.18077 & 0.14391 \\
\hline X64 & 1.6 & 0 & 1.5 & 1.26195 & 0.14745 \\
\hline X65 & 1.6 & 0.025 & 1.5 & 1.27096 & 0.14797 \\
\hline X66 & 1.6 & 0.05 & 1.5 & 1.27995 & 0.14848 \\
\hline X67 & 2.0 & 0 & 1.5 & 1.47042 & 0.15624 \\
\hline $\mathrm{X68}$ & 2.0 & 0.025 & 1.5 & 1.48129 & 0.15679 \\
\hline $\begin{array}{l}\mathrm{X69} \\
\end{array}$ & 2.0 & 0.05 & 1.5 & 1.49217 & 0.15735 \\
\hline X70 & 3.0 & 0 & 1.5 & 2.03655 & 0.17598 \\
\hline X71 & 3.0 & 0.025 & 1.5 & 2.05329 & 0.17604 \\
\hline X72 & 3.0 & 0.05 & 1.5 & 2.07002 & 0.17669 \\
\hline X73 & 5.0 & 0 & 1.5 & 3.24417 & 0.20237 \\
\hline X74 & 5.0 & 0.025 & 1.5 & 3.27708 & 0.20318 \\
\hline X75 & 5.0 & 0.05 & 1.5 & 3.30999 & 0.20400 \\
\hline X76 & 10.0 & 0 & 1.5 & 6.36472 & 0.23245 \\
\hline X77 & 10.0 & 0.025 & 1.5 & 6.46101 & 0.23370 \\
\hline X78 & 10.0 & 0.05 & 1.5 & 6.55830 & 0.23494 \\
\hline
\end{tabular}




\begin{tabular}{|c|c|c|c|c|c|}
\hline $\mathrm{U}$ & $\mathrm{M}$ & $\mathrm{K}_{0}$ & $\Delta$ & $f^{\prime \prime}(0)$ & $-g^{\prime}(0)$ \\
\hline X79 & 0.0 & 0 & 5 & 0.47168 & 0.25896 \\
\hline X80 & 0.0 & 0.025 & 5 & 0.47284 & 0.25933 \\
\hline X81 & 0.0 & 0.05 & 5 & 0.47400 & 0.25970 \\
\hline X82 & 0.2 & 0 & 5 & 0.47765 & 0.26071 \\
\hline X83 & 0.2 & 0.025 & 5 & 0.47882 & 0.26108 \\
\hline X84 & 0.2 & 0.05 & 5 & 0.48000 & 0.26146 \\
\hline X85 & 0.4 & 0 & 5 & 0.49521 & 0.26581 \\
\hline X86 & 0.4 & 0.025 & 5 & 0.49642 & 0.26619 \\
\hline X87 & 0.4 & 0.05 & 5 & 0.49764 & 0.26657 \\
\hline X88 & 0.6 & 0 & 5 & 0.52346 & 0.27384 \\
\hline X89 & 0.6 & 0.025 & 5 & 0.52474 & 0.27423 \\
\hline X90 & 0.6 & 0.05 & 5 & 0.52602 & 0.27462 \\
\hline X91 & 0.8 & 0 & 5 & 0.56108 & 0.28425 \\
\hline X92 & 0.8 & 0.025 & 5 & 0.56244 & 0.28465 \\
\hline X93 & 0.8 & 0.05 & 5 & 0.56381 & 0.28506 \\
\hline X94 & 1.0 & 0 & 5 & 0.60662 & 0.29642 \\
\hline X95 & 1.0 & 0.025 & 5 & 0.60809 & 0.29684 \\
\hline X96 & 1.0 & 0.05 & 5 & 0.60957 & 0.29726 \\
\hline X97 & 1.2 & 0 & 5 & 0.65865 & 0.30977 \\
\hline X98 & 1.2 & 0.025 & 5 & 0.66025 & 0.31021 \\
\hline X99 & 1.2 & 0.05 & 5 & 0.66186 & 0.31065 \\
\hline X100 & 1.4 & 0 & 5 & 0.71593 & 0.32383 \\
\hline X101 & 1.4 & 0.025 & 5 & 0.71768 & 0.32429 \\
\hline X102 & 1.4 & 0.05 & 5 & 0.71943 & 0.32475 \\
\hline X103 & 1.6 & 0 & 5 & 0.77738 & 0.33821 \\
\hline X104 & 1.6 & 0.025 & 5 & 0.77929 & 0.33869 \\
\hline X105 & 1.6 & 0.05 & 5 & 0.78121 & 0.33917 \\
\hline X106 & 2.0 & 0 & 5 & 0.90960 & 0.36692 \\
\hline X107 & 2.0 & 0.025 & 5 & 0.91188 & 0.36745 \\
\hline X108 & 2.0 & 0.05 & 5 & 0.91417 & 0.36797 \\
\hline X109 & 3.0 & 0 & 5 & 1.27182 & 0.43286 \\
\hline X110 & 3.0 & 0.025 & 5 & 1.27525 & 0.43350 \\
\hline X111 & 3.0 & 0.05 & 5 & 1.27868 & 0.43414 \\
\hline X112 & 5.0 & 0 & 5 & 2.05015 & 0.53342 \\
\hline X113 & 5.0 & 0.025 & 5 & 2.05667 & 0.53428 \\
\hline X114 & 5.0 & 0.05 & 5 & 2.06318 & 0.53514 \\
\hline X115 & 10.0 & 0 & 5 & 4.06866 & 0.67589 \\
\hline X116 & 10.0 & 0.025 & 5 & 4.08709 & 0.67718 \\
\hline X117 & 10.0 & 0.05 & 5 & 4.10552 & 0.67848 \\
\hline
\end{tabular}


International Journal of Engineering Research and Technology. ISSN 0974-3154, Volume 13, Number 1 (2020), pp. 170-180

(C) International Research Publication House. https://dx.doi.org/10.37624/IJERT/13.1.2020.170-180

Then applying the proposed approach based on rough set theory which summaries as:

Step 1: discretize the decision table by using the Boolean reasoning algorithm.

Step 2: compute the reduct of the discretized decision table with the aid of genetic algorithm.

Step 3: generate a set of maximally generalized decision rules (classification rules).

The following flowchart represents the complete steps to extract the set of classification rules.

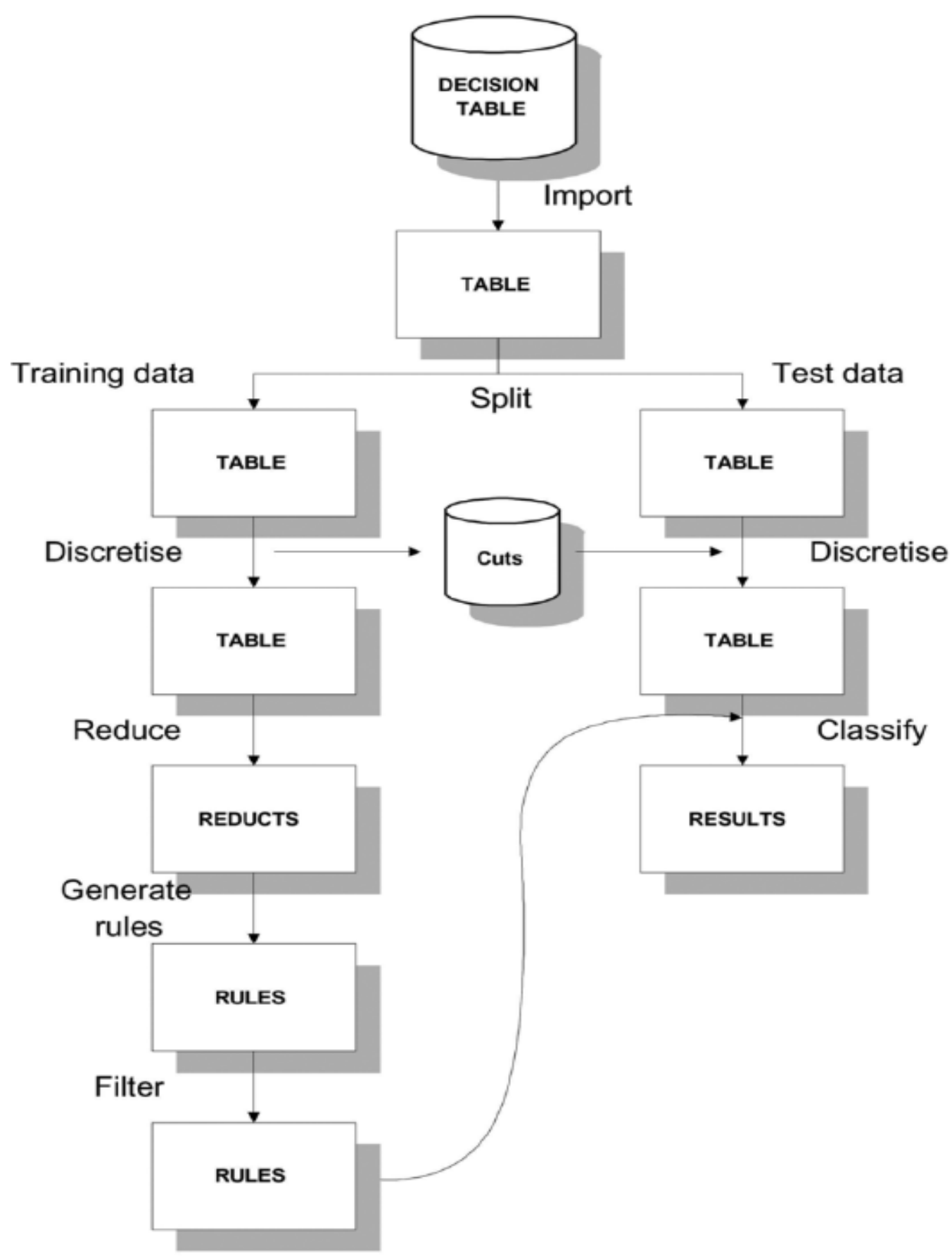

Fig. 2: Complete Steps to Extract Decision Rules

It worth noting that in this stage we will use software called ROSETTA which is an RST analysis toolkit. Table 3 shows part of the rule set extracted by using rough set methodology which explained in fig. 2. 


\begin{tabular}{|c|}
\hline Rules \\
\hline$\vdots$ \\
\hline IF $\left(M=[0.7,0.9) \wedge \mathrm{K}_{0}=[*, 0.013)\right) \operatorname{THEN}\left(\mathrm{f}^{\prime \prime}(0)=\{0.49265\}\right)$ \\
\hline $\mathrm{IF}\left(M=[2.5,4.0) \wedge \mathrm{K}_{0}=[0.013,0.038)\right) \mathrm{THEN}\left(\mathrm{f}^{\prime \prime}(0)=\{3.33951\}\right)$ \\
\hline IF $\left(M=[4.0,7.5) \wedge \mathrm{K}_{0}=[*, 0.013)\right) \operatorname{THEN}\left(\mathrm{f}^{\prime \prime}(0)=\{5.14796\}\right)$ \\
\hline $\mathrm{IF}\left(M=[7.5, *) \wedge \mathrm{K}_{0}=[0.013,0.038)\right) \mathrm{THEN}\left(\mathrm{f}^{\prime \prime}(0)=\{10.63314\}\right)$ \\
\hline $\mathrm{IF}\left(M=[7.5, *) \wedge \mathrm{K}_{0}=[0.038, *)\right) \mathrm{THEN}\left(\mathrm{f}^{\prime \prime}(0)=\{11.25077\}\right)$ \\
\hline $\mathrm{IF}\left(M=[0.5,0.7) \wedge \mathrm{K}_{0}=[*, 0.013) \wedge \Delta=[*, 1.0)\right) \mathrm{THEN}\left(-\mathrm{g}^{\prime}(0)=\{0.04959\}\right)$ \\
\hline $\mathrm{IF}\left(M=[0.7,0.9) \wedge \mathrm{K}_{0}=[0.038, *) \wedge \Delta=[*, 1.0)\right) \mathrm{THEN}\left(-\mathrm{g}^{\prime}(0)=\{0.05140\}\right)$ \\
\hline $\mathrm{IF}\left(M=[0.5,0.7) \wedge \mathrm{K}_{0}=[0.038, *) \wedge \Delta=[3.3, *)\right) \mathrm{THEN}\left(-\mathrm{g}^{\prime}(0)=\{0.27462\}\right)$ \\
\hline $\mathrm{IF}\left(M=[7.5, *) \wedge \mathrm{K}_{0}=[0.013,0.038) \wedge \Delta=[3.3, *)\right) \mathrm{THEN}(-\mathrm{g}(0)=\{0.67718\})$ \\
\hline IF $\left(M=[0.1,0.3) \wedge \mathrm{K}_{0}=[0.013,0.038) \wedge \Delta=[*, 1.0)\right) \mathrm{THEN}\left(\mathrm{f}^{\prime \prime}(0)=\{1.03056\}\right)$ \\
\hline $\mathrm{IF}\left(M=[0.1,0.3) \wedge \mathrm{K}_{0}=[0.038, *) \wedge \Delta=[3.3, *)\right) \mathrm{THEN}\left(\mathrm{f}^{\prime \prime}(0)=\{0.48000\}\right)$ \\
\hline$\vdots$ \\
\hline
\end{tabular}

It is noted that In the case of viscoelastic fluids $f^{\prime \prime}(0)$ is proportional to the friction factor. And In the case of micropolar viscoelastic fluids $f^{\prime \prime}(0)$ is proportional to the friction factor and $g^{\prime}(0)$ is proportional to the wall couple stress.

\section{CONCLUSION}

This paper suggests the use of rough set theory to process and extract rules for Analyzing the Effect of Viscoelastic and Micropolar Parameters on Hiemenz Flow in Hydromagnetics. The results of this study indicate that as the micropolar parameter $\Delta$ increases, the friction factor decreases. A similar behaviour is noted when the Hartman number and the viscoelastic parameter increase. The absolute value of the microrotation gradient is found to increase with increasing Hartman number, micropolar parameter, and viscoelastic parameter. Also the obtained results are in good agreement with previous studies. The technique has been simplified logic-based rules, reduces the time and resources required to building knowledge.

\section{ACKNOWLEDGMENT}

The author thank Prince Sattam bin Abdulaziz University, Deanship of Scientific Research at Prince Sattam bin Abdulaziz University for their continuous support and encouragement.

\section{REFERENCES}

[1] Beard, D. W., and Ken Walters. "Elastico-viscous boundary-layer flows I. Two-dimensional flow near a stagnation point." Mathematical Proceedings of the Cambridge Philosophical Society. Vol. 60. No. 3. Cambridge University Press, 1964. 
[2] Walters, Kj. "Non-Newtonian effects in some elasticoviscous liquids whose behaviour at small rates of shear is characterized by a general linear equation of state." The Quarterly Journal of Mechanics and Applied Mathematics 15.1 (1962): 63-76.

[3] Bhattacharyya, S., A. Pal, and A. S. Gupta. "Heat transfer in the flow of a viscoelastic fluid over a stretching surface." Heat and mass transfer 34.1 (1998): 41-45.

[4] Nabwey, Hossam A., and Hamed A. El-Mky. "Lie group analysis of thermophoresis on a vertical surface in a porous medium." Journal of King Saud UniversityScience 31, no. 4 (2019): 1048-1055.

[5] Abel, M. Subhas, and N. Mahesha. "Heat transfer in MHD viscoelastic fluid flow over a stretching sheet with variable thermal conductivity, non-uniform heat source and radiation." Applied Mathematical Modelling 32.10 (2008): 1965-1983.

[6] Nabwey, Hossam A., S. M. M. EL-Kabeir, and A. M. Rashad. "Lie group analysis of effects of radiation and chemical reaction on heat and mass transfer by unsteady slip flow from a non-isothermal stretching sheet immersed in a porous medium." Journal of Computational and Theoretical Nanoscience 12, no. 11 (2015): 4056-4062.

[7] Animasaun, I. L., C. S. K. Raju, and N. Sandeep. "Unequal diffusivities case of homogeneousheterogeneous reactions within viscoelastic fluid flow in the presence of induced magnetic-field and nonlinear thermal radiation." Alexandria Engineering Journal 55.2 (2016): 1595-1606.

[8] Nabwey, Hossam A., Mohamed Boumazgour, and A. M. Rashad. "Group method analysis of mixed convection stagnation-point flow of non-Newtonian nanofluid over a vertical stretching surface." Indian Journal of Physics 91, no. 7 (2017): 731-742.

[9] Raju, V. Naga, K. Hemalatha, and V. Srihari Babu. "MHD Viscoelastic Fluid Flow Past an Infinite Vertical Plate in the Presence of Radiation and Chemical Reaction." International Journal of Applied Engineering Research 14.5 (2019): 1062-1069.

[10] Ingelsten, Simon, Andreas Mark, and Fredrik Edelvik. "A Lagrangian-Eulerian framework for simulation of transient viscoelastic fluid flow." Journal of NonNewtonian Fluid Mechanics 266 (2019): 20-32.

[11] Hiemenz, Karl. "Die Grenzschicht an einem in den gleichformigen Flussigkeitsstrom eingetauchten geraden Kreiszylinder." Dinglers Polytech. J. 326 (1911): 321-324.

[12] Li, Qing, et al. "Near-wall dynamics of a neutrallybuoyant particle in Hiemenz flow." Bulletin of the American Physical Society (2018).

[13] Bano, Nasreen, B. B. Singh, and S. R. Sayyed. "Homotopy Analysis For MHD Hiemenz Flow In a
Porous Medium With Thermal Radiation, Velocity and Thermal Slips Effects." Frontiers in Heat and Mass Transfer (FHMT) 10 (2018).

[14] Ghaffari, Abuzar, Irfan Mustafa, and Tariq Javed. "Time Dependent Convective Non-Orthogonal Hiemenz Flow of Viscoelastic Walter's B Fluid towards a Non-Uniformly Heated Vertical Surface: Using Spectral Method." Nihon Reoroji Gakkaishi 46.4 (2018): 155-164.

[15] Eringen, A. Cemal. "Theory of micropolar fluids." Journal of Mathematics and Mechanics (1966): $1-18$.

[16] Eringen, A. Cemal. "Linear theory of micropolar viscoelasticity." International Journal of Engineering Science5.2 (1967): 191-204.

[17] Arifuzzaman, S., et al. "Magnetohydrodynamic micropolar fluid flow in presence of nanoparticles through porous plate: A numerical study." International Journal of Heat and Technology 36.3 (2018): 936-948.

[18] Koriko, O. K., et al. "The combined influence of nonlinear thermal radiation and thermal stratification on the dynamics of micropolar fluid along a vertical surface." Multidiscipline Modeling in Materials and Structures 15.1 (2019): 133-155.

[19] Mehmood, Ammara, et al. "Integrated intelligent computing paradigm for the dynamics of micropolar fluid flow with heat transfer in a permeable walled channel." Applied Soft Computing (2019).

[20] Nabwey, Hossam A. "A Hybrid Approach for Extracting Classification Rules Based on Rough Set Methodology and Fuzzy Inference System and Its Application in Groundwater Quality Assessment." In Advances in Fuzzy Logic and Technology 2017, pp. 611-625. Springer, Cham, 2017.

[21] Nabwey, Hossam A., M. Modather, and M. Abdou. "Rough set theory based method for building knowledge for the rate of heat transfer on free convection over a vertical flat plate embedded in a porous medium." In 2015 International Conference on Computing, Communication and Security (ICCCS), pp. 1-8. IEEE, 2015.

[22] Nabwey, H.A.. An approach based on Rough Sets Theory and Grey System for Implementation of RuleBased Control for Sustainability of Rotary Clinker Kiln. International Journal of Engineering Research and Technology, Volume 12, Number 12 (2019), pp. 2604-2610

[23] Shaaban, Shaaban M., and H. Nabwey. "A decision tree approach for steam turbine-generator fault diagnosis." International Journal of Advanced Science and Technology 51 (2013): 59-66.

[24] Shaaban, Shaaban M., and Hossam A. Nabwey. "A probabilistic rough set approach for water reservoirs site location decision making." In International 
Conference on Computational Science and Its Applications, pp. 358-372. Springer, Berlin, Heidelberg, 2012.

[25] Shaaban, Shaaban M., and Hossam A. Nabwey. "Rehabilitation and reconstruction of asphalts pavement decision making based on rough set theory." In International Conference on Computational Science and Its Applications, pp. 316-330. Springer, Berlin, Heidelberg, 2012.

[26] Shaaban, M., and A. Nabwey. "Transformer fault diagnosis method based on rough set and generalized distribution table." Int J Intell Eng Syst 5 (2012): 1724.

[27] Mohamed, Hossam Abd Elmaksoud. "An Algorithm for Mining Decision Rules Based on Decision Network and Rough Set Theory." In International Conference on Ubiquitous Computing and Multimedia Applications, pp. 44-54. Springer, Berlin, Heidelberg, 2011.

[28] Zhao, Hong, Ping Wang, Qinghua Hu, and Pengfei Zhu. "Fuzzy Rough Set Based Feature Selection for Large-Scale Hierarchical Classification." IEEE Transactions on Fuzzy Systems 27, no. 10 (2019): 1891-1903.

[29] Nabwey, Hossam A., and Mahdy S. El-Paoumy. "An integrated methodology of rough set theory and grey system for extracting decision rules." International Journal of Hybrid Information Technology 6, no. 1 (2013): 57-65.

[30] Hu, Xiaoyuan, Bingzhen Sun, and Xiangtang Chen. "Double quantitative fuzzy rough set-based improved AHP method and application to supplier selection decision making." International Journal of Machine Learning and Cybernetics 11, no. 1 (2020): 153-167.

[31] Pathak, Hemant Kumar, Reny George, Hossam A. Nabwey, Mahdy S. El-Paoumy, and Kaivatath Puthalath Reshma. "Some generalized fixed point results in ab-metric space and application to matrix equations." Fixed Point Theory and Applications 2015, no. 1 (2015): 1-17.

[32] George, R., Nabwey, H.A., Reshma, K.P. and Rajagopalan, R., 2015. Generalized cone b-metric spaces and contraction principles. Mat. Vesn, 67(4), pp.246-257.

[33] El-Kabeir, S.M., 2005. Hiemenz flow of a micropolar viscoelastic fluid in hydromagnetics. Canadian journal of physics, 83(10), pp.1007-1017. 